\title{
Lunar periodicity of planula release in the reef-building coral Stylophora pistillata
}

\author{
David Zakai ${ }^{1,2, *}$, Zvy Dubinsky ${ }^{1}$, Amir Avishai ${ }^{1}$, Tamir Caaras ${ }^{1}$, \\ Nanette E. Chadwick ${ }^{1,3}$ \\ ${ }^{1}$ Faculty of Life Sciences, Bar Ilan University, Ramat Gan, Israel, and Interuniversity Institute for Marine Science, \\ PO Box 469, Eilat, Israel \\ ${ }^{2}$ Israel Nature \& Parks Authority, PO Box 667, Eilat, Israel \\ ${ }^{3}$ Present address: Department of Biological Sciences, 101 Rouse Life Science Building, Auburn University, Auburn, \\ Alabama 36849-5407, USA
}

\begin{abstract}
Stylophora pistillata is a dominant stony coral on reefs in the Red Sea, but the levels and periodicity of planula release in this coral are not well understood. We examined patterns of planula release by colonies under laboratory conditions in outdoor tanks at Eilat during 1998, then tagged and returned the corals to the adjacent reef. In 2003, we re-collected them and re-estimated their planula release patterns. In both years, the number of planula larvae released per night varied widely between colonies, and did not correlate with coral colony volume or diameter. Planula release rate per live coral volume was 5.6-fold lower in 2003 than in 1998, while coral volume increased 5.5-fold during this period. In both years, colonies of $S$. pistillata released planula larvae on a lunar cycle during the 4 mo of peak planulation (March to June). Peak release was on Night 6 following the full moon each month during 1998 and on Night 2 during 2003, with most planulation occurring between Nights 1 and 12. Collection of released planulae from randomly selected colonies of $S$. pistillata in the field during both years revealed the same lunar cycle of planula release as under laboratory conditions. In field colonies, the number of planulae released per coral was 3-fold lower in 2003 than in 1998. The observed reduction in planula release between years in the field parallels documented declines in coral recruitment and reef growth at Eilat, and may have resulted, in part, from anthropogenic impacts to local reefs. Added effects of coral aging in tagged experimental colonies may have caused the larger observed reduction in planula release observed under laboratory conditions. The proximate factors that regulate the above lunar periodicity of planulation are thought to include night irradiance patterns and tidal cycles, which also may be linked to gametogenesis cycles. Lunar periodicity ultimately may have developed to enhance the dispersal of released propagules during neap or spring tides, or to maximize fertilization of gametes through tight synchronization of coral reproduction among colonies.
\end{abstract}

KEY WORDS: Reproduction $\cdot$ Coral reef $\cdot$ Lunar cycle $\cdot$ Eilat $\cdot$ Red Sea $\cdot$ Colony size

\section{INTRODUCTION}

Variation in sexual reproductive characteristics among species of stony corals, especially in their patterns of synchronization and rates of propagule release, may strongly affect the persistence and community structure of coral reefs (Richmond 1997). The main environmental factors that regulate the annual reproductive cycles of corals are thought to be water temperature and day length, with fine tuning of monthly cycles controlled by lunar periodicity (reviewed in Harrison \& Wallace 1990, Richmond \& Hunter 1990, Richmond 1997). The timing of gamete release often varies geographically within a given coral species, and may reveal responses of populations to different environmental conditions (Oliver et al. 
1988, Harrison \& Wallace 1990, Richmond \& Hunter 1990, Babcock et al. 1994, Richmond 1997, Loya 2004). Levels of synchronization in planula release by pocilloporid corals are known to vary widely among locations (reviewed in Tanner 1996), but clear patterns have not yet emerged in terms of control by lunar and seasonal environmental factors (Harrison \& Wallace 1990, Shlesinger et al. 1998, Fautin 2002).

Colonies of the reef-building pocilloporid coral Stylophora pistillata dominate some Red Sea coral reef (Loya 1976b), and their patterns of sexual reproduction have been studied extensively in the 1970s and 1980s (Rinkevich \& Loya 1979a,b, 1986, 1987). In recent years the reproductive output of these corals at at Eilat has declined severely, possibly due to anthropogenic stress (Loya et al. 2004). At this site, S. pistillata colonies have an extended reproductive season of nightly planula release over about 8 mo each year (December to July), in contrast to other local corals that release sexual propagules during only a few nights each year, mainly in summer (Shlesinger \& Loya 1985). In large, old colonies of $S$. pistillata, planula release may decrease over several months prior to death, and the colony also may change sex to become male or sterile, indicating possible senescence (Rinkevich \& Loya 1986, Harrison \& Wallace 1990).

Sexual reproductive patterns of Stylophora pistillata colonies vary widely among locations in the Indo-Pacific region. At Heron Island in the southern Great Barrier Reef (GBR), S. pistillata planulates only during summer, and planula presence inside polyps does not follow a lunar cycle (Tanner 1996). At Lizard Island in the northern GBR, S. pistillata also planulates only during the summer (Loya 1983), but its lunar periodicity has not been examined. In southern Taiwan, planula release by S. pistillata colonies occurs during both winter and summer months, with a monthly cycle of peak planula release on Lunar Days 6 to 7 following the full moon (i.e. Days 21 to 22 after the new moon; Fan et al. 2002). In nearby Palau, $S$. pistillata also exhibits lunar periodicity, with peak planula release between the full and new moons, and reproduction year-round (Atoda 1947). In contrast, in the Red Sea, it has been reported that planula release does not occur with lunar periodicity, and planulation occurs between December and July (winter to summer) (Rinkevich \& Loya 1979b, Shlesinger \& Loya 1985).

We re-examined here the question of lunar periodicity of planula release in Stylophora pistillata in the Red Sea, and documented changes in colony size and rates of planulation between years. We also compared the reproductive output of $S$. pistillata colonies under laboratory versus field conditions, to explore the reliability of laboratory culture methods for assessing reproductive output in stony corals.

\section{MATERIALS AND METHODS}

Laboratory observations. On December 12, 1997, 20 undamaged colonies of the branching coral Stylophora pistillata were selected haphazardly at 6 to $9 \mathrm{~m}$ depth on the coral reef adjacent to the Interuniversity Institute for Marine Science (IUI) near Eilat, Israel, northern Red Sea. Medium-sized colonies with diameters of 11 to $17 \mathrm{~cm}$ (i.e. 124 to $310 \mathrm{~cm}^{3}$ colony volume) were selected, because almost all colonies of this size are reproductively mature (Rinkevich \& Loya 1979a,b), but not yet senescent (Rinkevich \& Loya 1986). Each coral colony was placed in a $3 \mathrm{l}$ bowl on an outdoor water table located on the beach of the IUI. Each bowl received a continuous flow of unfiltered seawater at a rate of approximately $11 \mathrm{~min}^{-1}$ (after Jokiel 1985, Richmond 1985). Seawater was pumped from a depth of $4 \mathrm{~m}$, approximately $1 \mathrm{~m}$ above the coral reef, so that demersal zooplankton were included during their natural migrations above the reef (Alldredge \& King 1985). In this way, laboratory corals received a supply from the reef of planktonic food and other resources, especially during night hours when demersal zooplankton migrated up into the water column. The tables were covered with 2 layers of plastic net (mesh size: $1 \mathrm{~cm}^{2}$ ), so that light intensity (in microEinsteins, where $1 \mu \mathrm{E}=$ $1 \mu \mathrm{mol}$ of photons) under the net, as measured using a LI-COR model LI-185B light meter, ranged from 800 to $950 \mu \mathrm{E} \mathrm{m} \mathrm{m}^{-2} \mathrm{~s}^{-1}$, and equaled the light intensity at 6 to $9 \mathrm{~m}$ depth in the field (Shashar et al. 1993).

Each day for 92 d (March 13 to June 23, 1998), seawater temperature was measured in the wet table (range $=19.4$ to $24.5^{\circ} \mathrm{C}$, mean $=22.7^{\circ} \mathrm{C}, \mathrm{SD}=1.2^{\circ} \mathrm{C}, \mathrm{N}=$ 92 measurements) and in the field at a depth of $4 \mathrm{~m}$ adjacent to the IUI (location of laboratory seawater supply pump, range $=21.2$ to $24.0^{\circ} \mathrm{C}$, mean $=22.7^{\circ} \mathrm{C}$, $\mathrm{SD}=0.9^{\circ} \mathrm{C}, \mathrm{N}=92$ ) and also at $8 \mathrm{~m}$ depth (collection site of laboratory corals, range $=21.0$ to $23.9^{\circ} \mathrm{C}$, mean $=$ $22.5, \mathrm{SD}=0.65, \mathrm{~N}=92$ ). Although laboratory temperatures varied slightly more than did those in the field, they did not differ significantly from field seawater temperatures at either depth $\left(\mathrm{ANOVA}, F_{(2,273)}=0.01\right.$, $p=0.99)$. During the second phase of the project (March 17 to June 22, 2003), seawater temperature was measured only in the field at a depth of $4 \mathrm{~m}$ adjacent to the IUI (location of laboratory seawater supply pump, range $=21.3$ to $24.1^{\circ} \mathrm{C}$, mean $=22.3^{\circ} \mathrm{C}, \mathrm{SD}=$ $0.95^{\circ} \mathrm{C}, \mathrm{N}=98$ ), and did not differ significantly from temperatures measured 5 yr previously in the laboratory during 1998 (Student's $t$-test, $t=1.9, \mathrm{p}=0.99$ ). In 2003, laboratory temperatures were not compared with field temperatures, because no differences had been detected during the first year of the study.

Once each month, the bowls containing laboratory corals were cleaned of algae and sediments. Planula 
collector cups, constructed from plastic beakers and $150 \mu \mathrm{m}$ plankton mesh, were placed under the overflow of each bowl for the entire experimental period (modified after Jokiel 1985, Richmond 1985, Tanner 1996, McGuire 1998). During 7 wk of acclimation (December 12, 1997 to February 9, 1998), all of the experimental corals were observed to release planula larvae. This period corresponds to the beginning of the planulation season for Stylophora pistillata, when colonies begin to release mature planula larvae prior to the peak planulation season of March to May each year (Rinkevich \& Loya 1979b, 1987). Planula release was monitored from February 9, 1998, but colonies were not weighed until March 10 to avoid disturbing them. On March 10, 1998, at the beginning of the high-reproduction season for S. pistillata (Rinkevich \& Loya 1979a,b), the volume of each coral colony was measured using the water displacement method (Richmond 1985). All planulae released by each coral were collected from the bowls and collector cups, and were counted daily from February 9 to June 23, 1998, a period of 134 d. At the end of the experiment, the displaced volume of each coral colony was remeasured to assess changes in colony size. Corals then were tagged and attached to an underwater table at $10 \mathrm{~m}$ depth on the reef. In 2003, 5 yr later, all 20 corals were re-collected and the same parameters were re-measured in the laboratory using the methods above, during March 17 to June 22 (98 nights). In 2003, there was no acclimation period before planula collection in the laboratory, because corals were observed to planulate even during the acclimation period in 1998.

Field observations. In order to assess rates of natural planula release in the field by colonies of Stylophora pistillata, planula traps were constructed from plastic bottles, $150 \mu \mathrm{m}$ plankton mesh and funnels (Fig. 1). A funnel was positioned in each trap so that all planulae entering the bottle at the top of the trap were prevented from escaping. For most of 1 lunar cycle, in each of the 2 yr examined (May 13 to June 7, 1998 and June 10 to July 11, 2003), planula traps were installed each afternoon on 7 to 8 (in 1998) or 10 to 15 (in 2003) haphazardly chosen colonies of $S$. pistillata in the field. All traps were collected the following morning, and the collected planulae were removed and counted. By visiting different reef areas each night, different colonies were selected for sampling so that each field

colony was sampled only once. In order to sample field colonies that were similar to laboratory colonies, we sampled only undamaged colonies of 11 to $17 \mathrm{~cm}$ diameter at a depth of 6 to $8 \mathrm{~m}$ on the reef adjacent to the IUI. Totals of 204 (in 1998) and 258 (in 2003) S. pistillata colonies were sampled for planula output during the 26 (1998) and 25 (2003) nights of the field study each year. We also sampled planula release from 102 S. pistillata colonies (each 11 to $17 \mathrm{~cm}$ diameter) during June 10 to July 11,2003 at a depth of 10 to $12 \mathrm{~m}$ on the IUI reef. The number of planulae released by colonies at 10 to $12 \mathrm{~m}$ depth did not differ significantly from that of colonies located at 6 to $8 \mathrm{~m}$ depth ( $t$-test, $\mathrm{p}>0.05$, $\mathrm{N}_{(6-8 \mathrm{~m} \text { depth })}=258, \mathrm{~N}_{(10-12 \mathrm{~m} \text { depth })}=102$ corals $)$.

Statistical analyses. We applied Pearson correlation and Student's $t$-tests (Statistica, v6.0) to analyze relationships between planula release rate, coral size and year. Pearson correlations also were applied to examine the relationship between lunar cycles of planula release in laboratory versus field corals each year, and also between the number of laboratory colonies that planulated each night (that each released at least 1 planula) versus the total number of planulae released 
in the laboratory. For assessment of lunar periodicity patterns, we employed Rayleigh's test of circular statistics (Zar 1984), to determine whether planula release was distributed uniformly throughout the lunar cycle (after Tanner 1996, Fan et al. 2002). Periodicity of planula release was analyzed over 4 complete lunar cycles in 1998 (i.e. Cycles 1 to 4 for 1998, February 12 [full moon] to June 11 [full moon], $\mathrm{N}=120$ nights) and 3 lunar cycles in 2003 (i.e. Cycles 2 to 4 for 2003 [Cycle 1 not included because sampling began only in March], March 19 [full moon] to June 16 [full moon], $\mathrm{N}=90$ nights). Periodicity of planulation also was analyzed separately for the non-peak cycles in each year (Cycles 1, 3, 4 in 1998; Cycles 3 and 4 in 2003). Lunar periodicity in the following parameters was analyzed: the number of colonies that planulated each night, the total number of planula released each night and the number of planula released by high-output corals (those producing $>100$ planula per year, $\mathrm{N}=16$ ). Results are presented as means $( \pm 1$ SD) unless otherwise indicated.

\section{RESULTS}

\section{Numbers of planulae released and changes in colony size}

Planula release varied widely among coral colonies during both years in the laboratory (Table 1). Almost all released planulae ( 99\%) were found in the collector cups, but some were observed in the culture bowls and were counted as well. In 1998, the maximum number of planulae released by a single colony during 1 night was 288; this colony released a maximum of 5387 planulae over 134 nights and 5145 planulae over 98 nights during 1998. In 2003, the maximum number of planulae released by a single colony during 1 night was 407, and this colony released a maximum of 3475 planulae over 98 nights. For each coral, planula release also varied between years; total planula release by each coral in 2003 did not correlate with its release in 1998 over 98 congruent lunar nights (Pearson's correlation coefficient: $\mathrm{r}=-0.11, \mathrm{p}>0.05, \mathrm{~N}=20$ corals).
Within the small range of colony diameters examined here (11 to $17 \mathrm{~cm}$ in 1998 and 23 to $36 \mathrm{~cm}$ in 2003, $\mathrm{N}=20$ ), the number of planulae released per coral did not correlate with coral diameter (Pearson's correlation coefficient: $r=0.01, p>0.05$ for $1998 ; r=0.12, p>0.05$ for 2003) nor with coral colony volume in either of the 2 study years $(\mathrm{r}=0.15, \mathrm{p}>0.05$ for $1998 ; \mathrm{r}=0.17, \mathrm{p}>$ 0.05 for 2003).

In contrast to the above variation in planula release rates among the corals, rates of change in colony size varied little among the corals. Coral volume in 2003 was highly correlated with initial size in 1998 (Pearson's correlation coefficient: $\mathrm{r}=0.97, \mathrm{p}<0.05, \mathrm{~N}=20$ corals; Fig. 2). Coral colony volumes steadily increased throughout the $5 \mathrm{yr}$ study (range $=124$ to $310 \mathrm{~cm}^{3}$ and 11 to $17 \mathrm{~cm}$ diameter on March 11, 1998; 147 to $364 \mathrm{~cm}^{3}$ and 13 to $20 \mathrm{~cm}$ diameter on June 22, 1998; 624 to $1750 \mathrm{~cm}^{3}$ and 23 to $36 \mathrm{~cm}$ diameter on March 12, 2003; 709 to $1895 \mathrm{~cm}^{3}$ and 24 to $38 \mathrm{~cm}$ diameter during the final measurement on June 24, 2003). Change in coral colony volume during the laboratory period each year did not correlate with the total number of planulae they released, in terms of absolute change in volume

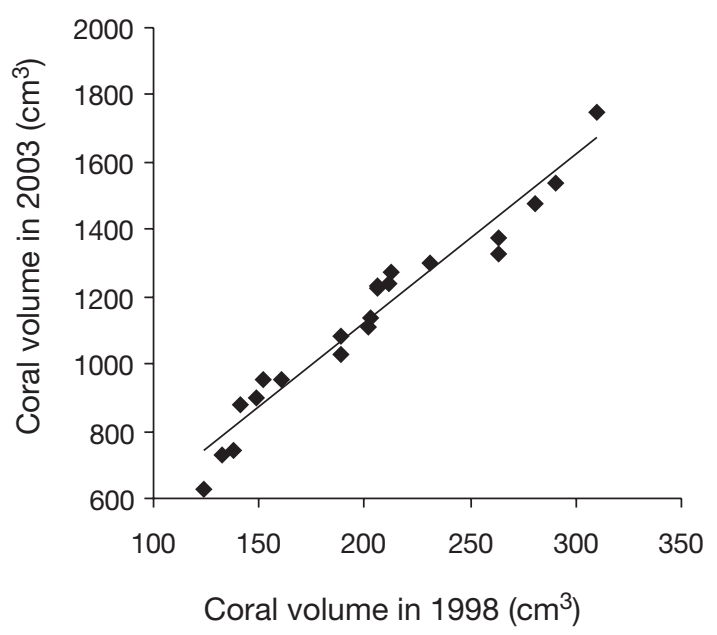

Fig. 2. Stylophora pistillata. Five-fold increase in the volume of colonies measured over $5 \mathrm{yr}$ (1998 to 2003) on the coral reef slope at $10 \mathrm{~m}$ depth at Eilat, northern Red Sea $(\mathrm{N}=20$ colonies). Each coral volume was measured on March 11, 1998, and then re-measured 5 yr later on March 12, 2003

Table 1. Stylophora pistillata. Number of planulae released in 1998 and 2003 by colonies of the reef-building coral under laboratory conditions. The same colonies $(\mathrm{N}=20)$ were observed during both years

\begin{tabular}{|c|c|c|c|c|c|c|c|c|c|}
\hline \multirow{3}{*}{ Year } & \multicolumn{2}{|l|}{ Chronological data } & \multicolumn{3}{|c|}{ Planula release ( $\mathrm{N}=20$ coral colonies) } & \multicolumn{4}{|c|}{ Number of colonies that released } \\
\hline & Date & No. nights & Per colony & Total & Range & & pla & ulae & \\
\hline & & & $(X \pm \mathrm{SD})$ & (all colonies) & per colony & $<100$ & $101-500$ & $501-2500$ & $>2500$ \\
\hline 1998 & 9 Feb-23 Jun & 134 & $1199 \pm 1501$ & 23977 & $14-5387$ & $4(20 \%)$ & $6(30 \%)$ & $7(35 \%)$ & $3(15 \%)$ \\
\hline 1998 & 11 Mar-16 Jun & 98 & $956 \pm 1230$ & 19120 & $6-5145$ & $4(20 \%)$ & $9(35 \%)$ & $6(30 \%)$ & $3(15 \%)$ \\
\hline 2003 & 17 Mar-22 Jun & 98 & $879 \pm 1033$ & 17578 & $16-3475$ & $5(25 \%)$ & $5(25 \%)$ & $8(40 \%)$ & $2(10 \%)$ \\
\hline
\end{tabular}


(Pearson's correlation coefficient: $\mathrm{r}=0.05, \mathrm{p}>0.05$ in $1998 ; \mathrm{r}=0.03, \mathrm{p}>0.05$ in 2003) or percent change $(\mathrm{r}=$ $-0.04, p>0.05$ in 1998; $r=-0.01, p>0.05$ in 2003).

Despite significantly larger coral volumes in 2003 versus 1998 (5.6-fold larger, $t$-test: $\mathrm{p}<0.0001, \mathrm{~N}=20$; Figs. $2 \& 3 \mathrm{~A}$ ), the number of planulae released per coral volume was significantly (5.5-fold) lower in 2003 than in 1998 ( $t$-test, $\mathrm{p}<0.005$, planula release compared for March 11 to June 16, 1998 versus March 17 to June 22, 2003; i.e. 96 nights in each year; Fig. 3B).

\section{Planula release in the laboratory versus field}

The number of planulae released per night by laboratory colonies correlated significantly with that by field colonies over a full lunar cycle in 1998 (Pearson's correlation coefficient: $\mathrm{r}=0.80, \mathrm{p}<0.05, \mathrm{~N}=26$; May 13 to June 7,1998$)$ and over part of a cycle in $2003(\mathrm{r}=$ $0.86, \mathrm{p}<0.05, \mathrm{~N}=11$ nights; June 10 to June 22, 2003). Laboratory and field sampling overlapped by only 11 nights in 2003, because laboratory sampling ended due to technical constraints (lack of manpower) before the end of the $25 \mathrm{~d}$ field sample (see 'Materials and methods' for details). Although laboratory and field colonies exhibited similar lunar patterns of planula release, during 1998 the laboratory colonies released $60 \%$ fewer planulae per colony than did field colonies (laboratory: $5.4 \pm 9.9$ planulae per colony per night, range $=1.8$ to $11.0, \mathrm{~N}=20$ corals; field: $9.0 \pm 11.0$ planulae per colony per night, range $=2.6$ to $33.7, \mathrm{~N}=204$ corals), and in 2003 the difference was even larger, with laboratory colonies releasing $95 \%$ fewer planulae per colony than did colonies sampled in the field (laboratory: $0.3 \pm 0.6$ planulae per colony per night, range $=$ 0 to $1.3, \mathrm{~N}=20$ corals; field: $5.5 \pm 9.4$ planulae per colony per night, range $=1.2$ to $17.0, \mathrm{~N}=123$ corals).

Planula release rates by field colonies also decreased significantly between years $\left(t\right.$-test, $\mathrm{p}<0.0001, \mathrm{~N}_{(1998)}=$ $141, \mathrm{~N}_{(2003)}=135$ corals sampled on 14 congruent lunar nights, May 23 to June 5, 1998 versus June 23 to July 7, 2003), and were on average 3-fold lower in 2003 than in 1998 (Fig. 3C).

\section{Periodicity of planula release}

Not all corals released planulae every night; the more corals that planulated during a given night, the greater the total number of planulae that were released that night in the laboratory. In both years, there was a significant correlation between the number of planulating corals and the total number of planulae released each night (Pearson's correlation coefficient: $\mathrm{r}=0.55, \mathrm{p}<0.001, \mathrm{~N}=134$ nights in 1998;
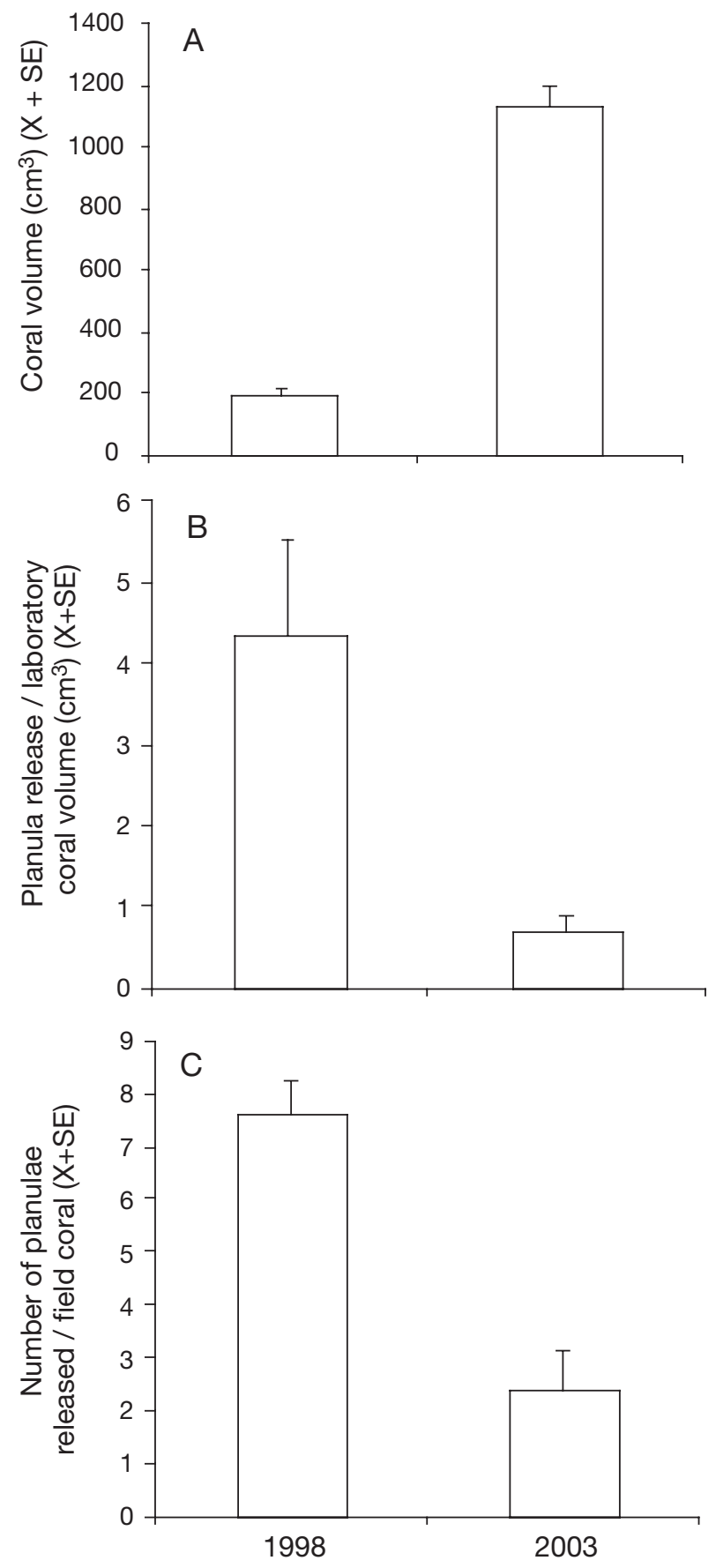

Fig. 3. Stylophora pistillata. Between-year variation in size and planula release patterns of colonies at Eilat, northern Red Sea. (A) Volume of colonies used in laboratory observations $(\mathrm{N}=20)$. During the $5 \mathrm{yr}$ between laboratory sessions, the corals were attached to an underwater table at $10 \mathrm{~m}$ depth on the coral reef. (B) Planula release per coral volume under laboratory conditions (mean $+\mathrm{SE}, \mathrm{N}=20$ colonies) during 98 congruent lunar nights in each year. (C) Number of planulae released from field colonies at 7 to $8 \mathrm{~m}$ depth on the coral reef. Data represent 14 congruent nights of sampling from May to June of each year examined ( $\mathrm{N}=141$ and 135 corals sampled in 1998 and 2003, respectively) 
$\mathrm{r}=0.67, \mathrm{p}<0.001, \mathrm{~N}=98$ nights in 2003). Both the number of colonies that planulated each night (Table 2A,B) and the total number of planulae released per night were distributed non-uniformly around the lunar cycle (Table 2C to H, Fig. 4).

In 1998, peak planula release occurred on Night 6 following each full moon (Figs. 4A \& 5A). More than half of all planulae (52\% of $\mathrm{N}=21799$ planulae; Table 2C) were released during Nights 2 to 11 following the full moon, for the 4 lunar cycles examined (February 12 to June 6, 1998), with a mean of $4.7 \%$ released per night. In contrast, only $35 \%$ of the planulae were released over a period twice as long, during Nights 15 to 30 (between the new and full moons), with a mean of $2.3 \%$ released per night. Peak planula production for the year occurred during Lunar Cycle 2
(March 13 to April 11, 1998), in which a large proportion of the planulae for that year were released $(44 \%$, $\mathrm{N}=21799 ;$ Table 2C). In contrast, only $22 \%$ were released during Cycle 1 (February 12 to March 12, 1998), 20\% during Cycle 3 (April 12 to May 10, 1998) and $14 \%$ in Cycle 4 (May 11 to June 6, 1998; Fig. 5A).

In 2003, peak planula release occurred on Night 2 following each full moon (Figs. 4B \& 5B). Again, more than half of all planulae (59\% of $\mathrm{N}=16969$ planulae Table 2G) were released during Nights 2 to 12 following the full moon, for the 3 lunar cycles examined (March 18 to June 13, 2003), with a mean of 5.3\% per night. In contrast, only $28.5 \%$ of the planulae were released over a period twice as long, during Nights 15 to 30 (between the new and full moons), with a mean of $1.9 \%$ released per night. As in 1998, peak planula pro-

Table 2. Stylophora pistillata. Test for uniformity of lunar distribution of the number of colonies that planulated and the number of planulae released by reef-building corals maintained in the laboratory at Eilat, northern Red Sea. $Z$ is the test statistic (Rayleigh's test, Zar 1984) and $\mathrm{N}$ is the number of colonies that planulated (A,B) or the number of planulae released (C-H). Lunar cycles in 1998 (1: February 12 to March 12; 2: March 13 to April 11; 3: April 12 to May 10; 4: May 11 to June 6) and in 2003 (2: March 18 to April 15; 3: April 16 to May 15; 4: May 16 to June 13). See 'Materials and methods' for details

\begin{tabular}{|lccccccc|}
\hline & $\begin{array}{c}\text { Lunar } \\
\text { cycles }\end{array}$ & Year & $\begin{array}{c}\text { Mean lunar } \\
\text { night }\end{array}$ & $\begin{array}{c}\text { Angular } \\
\text { deviation }\end{array}$ & $Z$ & N & $\begin{array}{c}\text { Probability of } \\
\text { uniform distribution }\end{array}$ \\
\hline $\begin{array}{l}\text { Colonies planulated } \\
\text { (A) }\end{array}$ & $2,3,4$ & 1998 & 5.5 & 6.5 & 3.7 & 90 & $0.02<\mathrm{p}<0.05$ \\
(B) & $2,3,4$ & 2003 & 8.9 & 6.4 & 7.8 & 90 & $<0.01$ \\
Planulae released & $1-4$ & 1998 & 6.4 & 5.8 & 1455 & 21799 & $<0.001$ \\
(C) & $1,2,3$ & 1998 & 6.8 & 6.3 & 163 & 11768 & $<0.001$ \\
(D) & $2,3,4$ & 1998 & 7.3 & 5.5 & 2047 & 17769 & $<0.001$ \\
(E) & 3,4 & 1998 & 7.2 & 6.1 & 232 & 7738 & $<0.001$ \\
(F) & $2,3,4$ & 2003 & 7.8 & 5.7 & 1439 & 16969 & $<0.001$ \\
(G) & 3,4 & 2003 & 9.1 & 6 & 305 & 5806 & $<0.001$ \\
(H) & & & & & & \\
\hline
\end{tabular}
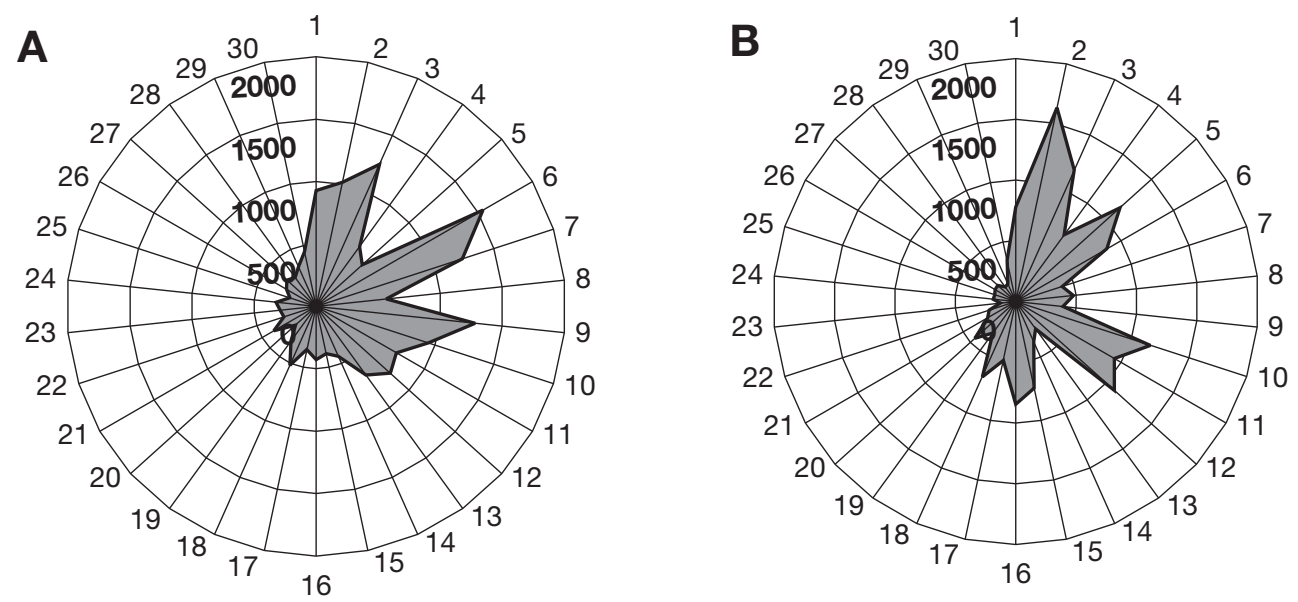

Fig. 4. Stylophora pistillata. Lunar periodicity of planula release by 20 colonies maintained in the laboratory at Eilat, northern Red Sea. Numbers in bold are axis labels representing the total number of planulae released each lunar night. Numbers around the circumference indicate the lunar night (Night 1 = full moon). Data were pooled from 3 lunar cycles during each of 2 years: (A) 1998 and (B) 2003 
duction was during Lunar Cycle 2 (March 18 to April $15,2003)$, in which the majority of planulae for that year were released (66\% of $\mathrm{N}=16969$ planulae; Table 2G), compared to only $27 \%$ during Cycle 3 (April 16 to May 15, 2003) and 7.3\% in Cycle 4 (May 16 to June 13, 2003; Fig. 5B).

Statistical analyses of the lunar periodicity of planula release during the non-peak cycles in each year (Cycles 1, 3 and 4 in 1998; Cycles 3 and 4 in 2003; Fig. 5) revealed periodicity even when the most prominent planula release cycle (No. 2) was removed from analysis (Table 2D,F,H). Each of the 16 high-producing colonies (that each released $>100$ planulae per year in 1998 and 2003) exhibited significant lunar periodicity of planula release (Rayleigh's test of circular statistics: $\mathrm{p}<0.05$ to 0.001 ). All of the high-producing corals peaked during the first half of each lunar cycle, between Nights 1 and 12, following the full moon (i.e. range of peak lunar nights for planula release, $\mathrm{N}=16$ colonies).

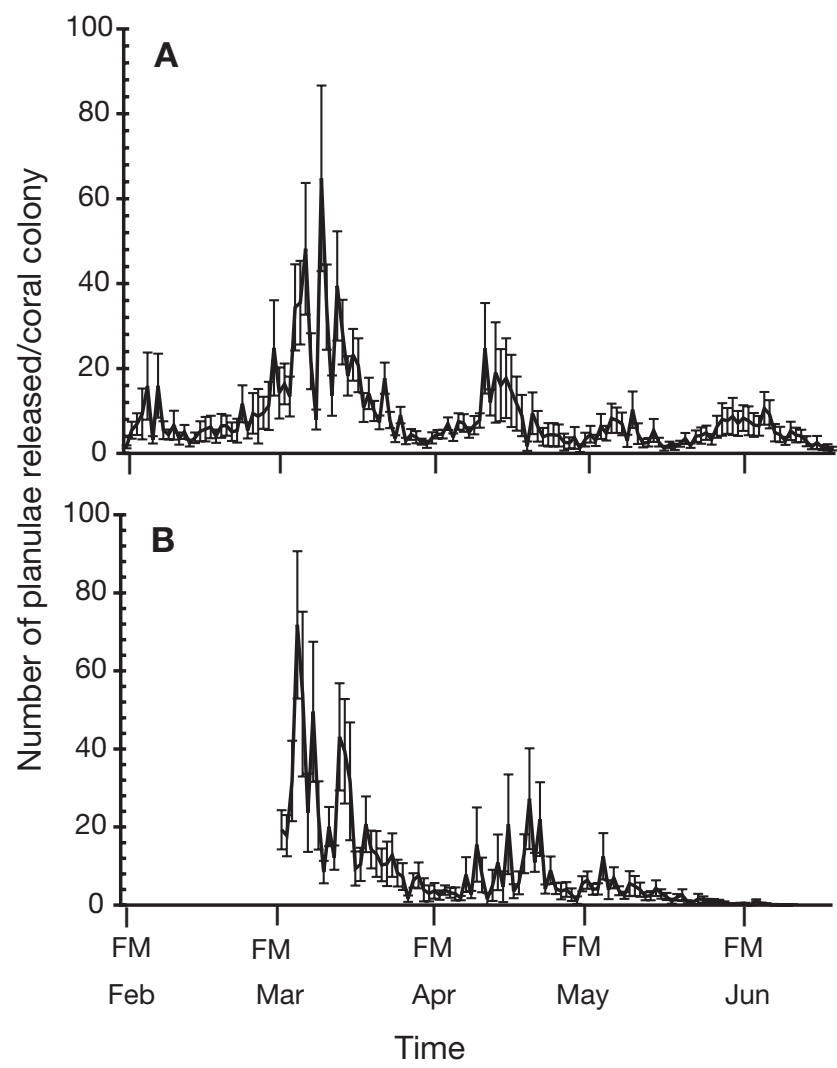

Fig. 5. Stylophora pistillata. Number of planulae released by coral colonies (mean $\pm \mathrm{SE}, \mathrm{N}=20$ ) under laboratory conditions at Eilat, northern Red Sea: (A) over 4 complete lunar cycles in 1998 (February 9 to June 24, 136 nights) and (B) over 3 complete lunar cycles in 2003 (March 17 to June 22, 98 nights). Between laboratory periods, the corals were maintained on an underwater table at $10 \mathrm{~m}$ depth on the coral reef. FM: full moon

\section{DISCUSSION}

The lower planula release rates of colonies under laboratory versus field conditions indicate that our laboratory culture methods affected reproduction in these corals, as has been observed for other coral species (McGuire 1998). Limited levels of water flow around the laboratory colonies may have inhibited gas exchange and, consequently, rates of coral growth and reproduction (reviewed in Goldshmid et al. 2004). Also, due to possible fouling of the seawater intake pipes by filter-feeding organisms, our laboratory corals likely received fewer zooplankton prey than did corals in the field. Fouling of the pipes also may have limited the supply of sperm reaching laboratory corals and, thus, lowered rates of gamete fertilization. However, planulation rates increased during several months in the laboratory (February to March; Fig. 5A) and paralleled those of field colonies, so corals did not appear to be severely limited by gamete fertilization. The March peak of planula release that we observed in Stylophora pistillata, with a gradual decline toward June and July (Fig. 5), has been described previously in the field at Eilat (Rinkevich \& Loya 1979b, 1987). Thus, the decline in planulation that we observed throughout each spring (Fig. 5) was probably not a laboratory artifact caused by sperm limitation. In spite of lower rates of planula output by laboratory versus field corals, they exhibited similar lunar patterns of output during the late spring (see 'Results'). Although reproductive rate may be reduced under laboratory conditions, periodicity of reproduction in this species can be detected by collecting released planula in the laboratory, as known for other coral species (Jokiel 1985, Richmond 1985, Tanner 1996, McGuire 1998). The accentuated difference between laboratory and field coral output during the second year of our study (Fig. 3B,C) may have been caused by aging and possible senescence of the older laboratory corals (Rinkevich \& Loya 1986, Harrison \& Wallace 1990). We did not observe effects of increased size and age between years in our fieldsampled colonies, because we selected different, medium-sized field colonies each year (see 'Materials and methods'). The large variation in planula release that we observed among colonies within each group is similar to that known from previous studies on $S$. pistillata (Rinkevich \& Loya 1979b) and other planulating corals (Harriott 1983, McGuire 1998).

Corals are known to reduce their fecundity due to environmental perturbations that cause sublethal stress (Harrison \& Wallace 1990). The large decrease in planula release between years that we observed in field-sampled corals (Fig. 3C) may have been due to natural variation (Rinkevich \& Loya 1987) 
and/or anthropogenically caused stress to coral reefs at Eilat (Loya 2004). The decrease in sexual reproduction that we observed here parallels recent declines in stony coral percent cover (Wielgus et al. 2003, Loya 2004), recruitment (Glassom et al. 2004) and calcification rates on coral reefs at Eilat (Silverman et al. 2004).

The lunar periodicity recorded here in the release of sexual propagules by colonies of Stylophora pistillata was similar to that known for many reef corals, in that peak release occurred each month following the full moon (reviewed in Harrison \& Wallace 1990, Richmond \& Hunter 1990, Richmond 1997, McGuire 1998). The pattern we observed here is most similar to that recorded previously for members of this species in Taiwan, in which planula release peaks on about Day 6 following the full moon (i.e. Day 21 after the new moon; Fan et al. 2002) (Figs. 4 \& 5). A similar pattern of lunar periodicity of planulation, peaking between the full and new moon, has been reported for $S$. pistillata colonies in Palau (Atoda 1947). Tanner (1996) also observed decreases in the number of planulae present inside $S$. pistillata polyps near full moon, but did not measure release rates. Unlike many other corals that release sexual propagules over a narrow range of only a few lunar days each month (Shlesinger \& Loya 1985, Babcock et al. 1986), $S$. pistillata colonies release planulae in a cycle that gradually rises to a peak and then declines, with low levels of release being maintained throughout the rest of the month (Fan et al. 2002) (Figs. 4 \& 5). This relatively scattered pattern of planula release during each lunar cycle has been noted for brooding corals (Harrison \& Wallace 1990), and may have contributed to the lack of periodicity reported in some previous studies on $S$. pistillata (Rinkevich \& Loya 1979b, Tanner 1996). Only daily measurement of planula release can detect lunar periodicity in these corals, in contrast to counting the planulae present inside the coral polyps.

Differences in both field and laboratory methods may have led to the different results in our study versus those by Rinkevich \& Loya $(1979 a$, b) on planulation patterns of Stylophora pistillata at Eilat. Laboratory methods in the latter studies consisted of histological sectioning to assess the maturation stages of planulae present inside the coral polyps. Mature planulae of the pocilloporid corals S. pistillata, Seriatopora hystrix and Pocillopora damicornis may be held inside the polyp from one reproductive season to another (i.e. from one year to the next; Tanner 1996). Thus, observations of planulae inside polyps can indicate release patterns only if there is a clear cycle of planula maturation (as in Atoda 1947). If no such cycle is apparent (as in Rinkevich \& Loya 1979b, Tanner 1996), then the only way to confirm an absence of planulation periodicity is by counting how many of these planulae are actually released each night by the corals, as was done in the present laboratory study. Due to the initially positive buoyancy of $S$. pistillata larvae (see below), our outflow traps captured almost all of the larvae released each night by each colony (see 'Results'), as has been demonstrated for other brooding corals (Jokiel 1985, Richmond 1985).

Field methods for planula collection may have prevented detection of periodicity in the past. In our field studies, when planula larvae were released by corals at night, most of them initially floated to the tops of the collection bottles (Fig. 1) that were positioned above the parent corals. When the traps were removed from the reef the next morning, most of the planulae then were observed near the bottom of each bottle. Thus, upon initial release the planulae of Stylophora pistillata appear to be positively buoyant, and only later in the night do they become negatively buoyant, similar to the sexual propagules of many corals (Harrison \& Wallace 1990). Our funnel device (Fig. 1), thus, prevents sinking larvae from leaving the bottle trap and settling to the bottom of the net near the coral base. Previous work on planulation patterns in S. pistillata at Eilat (Rinkevich \& Loya 1979b) deployed nets that lacked funnels, during the first half of each night. This method may have failed to detect some of the negatively buoyant larvae occurring near the net base, where they could potentially escape when the net was removed from the parent coral ${ }_{i}$ it also excluded any larvae released later during the night.

Why do Stylophora pistillata corals planulate on a lunar cycle? Several environmental cues are thought to control reproductive cycles in corals (e.g. temperature, day length; Harrison \& Wallace 1990), but only a few of these vary with the lunar cycle and so can be implicated in the precise timing of larval release each month. The proximate environmental cue for lunar cycling may be night irradiance, which has been demonstrated to control planulation cycles in Pocillopora damicornis (Jokiel et al. 1985). Tidal cycles may also contribute to control of lunar periodicity of planulation in corals, but this has not yet been clearly demonstrated (reviewed in Harrison \& Wallace 1990). Lunar periodicity of planula release in stony corals may result from lunar cycling of gametogenesis and fertilization rather than some cue for the larval release itself (Szmant-Froelich et al. 1985, McGuire 1998, Fan et al. 2002). This may, in part, explain why planula release is less tightly synchronized and is more scattered over the lunar cycle than is gamete spawning in corals, because some asynchrony likely develops following fertilization as the brooded larvae mature. Addition- 
ally, since some larvae may be retained within polyps after they mature (Tanner 1996), further asynchrony is introduced. Ultimately, the factors that cause the lunar cycling of planula release may be related to the advantages of release on neap or spring tides, which can increase the dispersal rates of coral larvae (Babcock et al. 1986, 1994, Oliver et al. 1988). Finally, since synchronization of larval release in corals may be a byproduct or side effect of synchronized gamete maturation, an ultimate cause of cycling in larval release may be the high levels of fertilization achieved by periodicity in gamete maturation (reviewed in Harrison \& Wallace 1990).

The timing of planula release in pocilliporid corals does not yet appear to follow clear patterns among geographic regions, and more populations and species need to be sampled in order to generate comparative data. In addition, rigorous methods need to be applied to detect lunar cycles of planulation in brooding corals. It is likely that more populations will be found to show lunar cycling as efforts are increased to accurately sample planula release by stony corals under field and laboratory conditions.

Acknowledgements. We thank the staff of the Interuniversity Institute for Marine Science in Eilat for providing logistical support throughout this study. Numerous volunteers assisted in the field and laboratory work, especially B. George and J. Stothard. We also thank the Israel Nature and Parks Authority for support and for Working Permits 8795-1998 and 1652-2003. Funding was provided, in part, by grants from the Research Authority of Bar Ilan University and from the Red Sea Marine Peace Park Program (RSMPP) of the United States Agency for International Development-Middle Eastern Regional Cooperation (USAIDMERC) to N.E.C.

\section{LITERATURE CITED}

Alldredge AL, King JL (1985) The distance demersal zooplankton migrate above the benthos: implications for predation. Mar Biol 84:253-260

Atoda K (1947) The larvae and postlarval development of some reef-building corals. II. Stylophora pistillata (Esper). Sci Rep Tohoku Univ 4 Ser Biol 18:48-65

Babcock RC, Bull GD, Harrison PL, Heyward AJ, Oliver JK, Wallace CC, Willis BL (1986) Synchronous spawning of 105 scleractinian coral species on the Great Barrier Reef. Mar Biol 90:379-394

Babcock RC, Wills BL, Simpson CJ (1994) Mass spawning of corals on a high latitude coral reef. Coral Reefs 13:161-169

Fan TY, Li JJ, Ie SX, Fang LS (2002) Lunar periodicity of larval release by pocilloporid corals in southern Taiwan. Zool Stud 41:288-294

Fautin DG (2002) Reproduction of Cnidaria. Can J Zool 80: $1735-1754$

Glassom D, Zakai D, Chadwick-Furman NE (2004) Are coral recruitment patterns consistent? A spatio-temporal analysis along the coastline of Eilat, northern Red Sea. Mar Biol 144:641-651
Goldshmid R, Holzman R, Weihs D, Genin A (2004) Aeration of corals by sleep-swimming fish. Limnol Oceanogr 49: 1832-1839

Harriott VJ (1983) Reproductive seasonality, settlement, and post-settlement mortality of Pocillopora damicornis (Linnaeus) at Lizard Island, Great Barrier Reef. Coral Reefs 2: 151-157

Harrison PL, Wallace CC (1990) Reproduction, dispersal and recruitment of scleractinian corals. In: Dubinsky Z (ed) Ecosystems of the world: coral reefs. Elsevier Science, Amsterdam, p 133-207

Jokiel PL (1985) Lunar periodicity of planula release in the reef coral Pocillopora damicornis in relation to various environmental factors. In: Gabrié C, Harmeli V (eds) Proc 5th Int Coral Reef Congr, Antenne Museum-EPHE, Moorea, Tahiti, 4:307-312

Jokiel PL, Ito RY, Liu PM (1985) Night irradiance and synchronization of lunar release of planula larvae in the reef coral Pocillopora damicornis. Mar Biol 88:167-174

Loya Y (1976) The Red Sea coral Stylophora pistillata is an r-strategist. Nature 259:478-480

Loya Y (1983) Reproduction strategy of Stylophora pistillata in the Great Barrier Reef and the Red Sea. In: Baker JT, Carter RM, Sammarco PW, Stark KP (eds) Proceedings of the inaugural Great Barrier Reef conference. James Cook University Press, Townsville, p 229

Loya Y (2004) The coral reefs of Eilat-past, present and future: three decades of coral community structure studies. In: Rosenberg E, Loya Y (eds) Coral health and disease. Springer, Berlin, p 1-34

Loya Y, Lubinevsky $H$, Rosenfeld M, Kramarsky-Winter E (2004) Nutrient enrichment caused by in situ fish farms at Eilat, Red Sea is detrimental to coral reproduction. Mar Pollut Bull 49:344-353

McGuire MP (1998) Timing of larval release by Porites astreoides in the northern Florida Keys. Coral Reefs 17: 369-375

Oliver JK, Babcock RC, Harrison PL, Willis BL (1988) Geographic extent of mass coral spawning: clues to ultimate causal factors. In: Choat $\mathrm{JH}$, Barnes D, Borowitzka MA, Coll JC and 14 others (eds) Proc 6th Int Coral Reef Symp, Vol 2. Symposium Executive Committee, Townsville, p 853-859

Richmond RH (1985) Variations in the population biology of Pocillopora damicornis across the Pacific. In: Gabrié C, Harmeli V (eds) Proc 5th Int Coral Reef Congr, Antenne Museum -EPHE, Moorea, Tahiti, 6:101-106

Richmond RH (1997) Reproduction and recruitment in corals: critical links in the persistence of reefs. In: Birkeland C (ed) Life and death of coral reefs. Chapman \& Hall, New York, p 175-197

Richmond RH, Hunter CL (1990) Reproduction and recruitment of corals: comparisons among the Caribbean, the tropical Pacific, and the Red Sea. Mar Ecol Prog Ser 60: 185-203

Rinkevich B, Loya Y (1979a) The reproduction of the Red Sea coral Stylophora pistillata. I. Gonads and planulae. Mar Ecol Prog Ser 1:133-144

Rinkevich B, Loya Y (1979b) The reproduction of the Red Sea coral Stylophora pistillata. II. Synchronization in breeding and seasonality of planulae shedding. Mar Ecol Prog Ser $1: 145-152$

Rinkevich B, Loya Y (1986) Senescence and dying signals in a reef building coral. Experientia 42:320-322

Rinkevich B, Loya Y (1987) Variability in the pattern of sexual reproduction of the coral Stylophora pistillata at Eilat, Red Sea: a long term study. Biol Bull (Woods Hole) 173:335-344 
Shashar N, Cohen Y, Loya Y (1993) Extreme diel fluctuations of oxygen in diffusive boundary layers surrounding stony corals. Biol Bull (Woods Hole) 185:455-461

Shlesinger Y, Loya Y (1985) Coral community reproductive patterns: Red Sea versus the Great Barrier Reef. Science 228:1333-1335

Shlesinger Y, Goulet TL, Loya Y (1998) Reproductive patterns of scleractinian corals in the northern Red Sea. Mar Biol 132:691-701

Silverman J, Lazar B, Erez J (2004) Monitoring the health of coral reef ecosystems using community metabolism. In: Rosenberg E, Loya Y (eds) Coral health and disease. Springer, Berlin, p 367-373

Editorial responsibility: Otto Kinne (Editor-in-Chief), Oldendorf/Luhe, Germany
Szmant-Froelich A, Reutter M, Riggs L (1985) Sexual reproduction of Favia fragum (Esper): lunar patterns of gametogenesis, embryogenesis and planulation in Puerto Rico. Bull Mar Sci 37:880-892

Tanner JE (1996) Seasonality and lunar periodicity in the reproduction of pocilloporid corals. Coral Reefs 15:59-66

Wielgus J, Glassom D, Fishelson L (2003) Long-term persistence of low coral cover and abundance on a disturbed coral reef flat in the northern Red Sea. Mar Pollut Bull 48: $248-253$

Zar JH (1984) Biostatistical analysis. Prentice-Hall, Englewood Cliffs, NJ

Submitted: February 14, 2005; Accepted: November 10, 2005 Proofs received from author(s): March 31, 2006 